\title{
MARKETING COMO FERRAMENTA DE TRABALHO DOS FUTUROS PROFISSIONAIS DE EDUCAÇÃO FÍSICA
}

\author{
Vilcor André da Cruz Anhanha ${ }^{1}$ \\ Marília de Rosso $\mathrm{Krug}^{2}$ \\ Vânia Mari Rossato ${ }^{3}$ \\ Rodrigo de Rosso $\mathrm{Krug}^{4}$
}

\begin{abstract}
RESUMO
No ramo da Educação Física muitas pessoas empreendem e utilizam de ferramentas do marketing para alavancar seus negócios, ainda mais em momentos em que vivemos com a pandemia, um cenário que automaticamente obriga o profissional a se reinventar. Neste sentido, este estudo teve como objetivo analisar como a temática marketing digital está sendo estudada, percebida e desenvolvida por acadêmicos de Educação Física Bacharelado. Esta pesquisa qualitativa descritiva contou com a participação de 10 acadêmicos do $8^{\circ}$ semestre do referido curso. A coleta de dados se deu por meio de um "questionário digital" através da ferramenta Google formulários interpretados por análise de conteúdo. Sete destes acadêmicos disseram que o curso não ofereceu uma boa formação na temática e indicaram ser necessário mais disciplinas que abordem sobre marketing tendo em vista que todos os participantes relataram que somente a disciplina de Marketing abordou sobre o tema. Em relação ao seu conhecimento sobre o marketing, a maioria, respondeu que possuíam conhecimento básico. Ainda em relação ao conhecimento sobre a temática em questão, todos acadêmicos responderam que, se já fossem formados, utilizariam como estratégias de marketing as redes sociais divulgando vídeos e postagens, além de aulas on-line. Todos os acadêmicos relataram que conhecimentos sobre marketing são de extrema importância para a profissão. Conclui-se que o marketing está sendo abordado e utilizado de maneira tímida pelos futuros profissionais de Educação Física pesquisados, sendo necessário um maior investimento deles na área.
\end{abstract}

PALAVRAS-CHAVE: educação física. marketing. empreendedorismo. formação inicial.

\begin{abstract}
ABSCTRAT
In the field of Physical Education many people undertake and use marketing tools to leverage their business, especially in times when we live with the pandemic, a scenario that automatically forces the professional to reinvent themselves. In this sense, this study aimed to analyze how the digital marketing theme is being studied, perceived and developed by Academics of Physical Education Bachelor's Degree. This descriptive qualitative research had the participation of 10 students from the 8 th semester of this course. The data collection took place through a "digital questionnaire" developed with the Google forms tool interpreted by content analysis. Seven of these students said that the course did not offer a good training in the theme and indicated that more disciplines were needed to address marketing, considering that all participants reported that only the marketing course addressed the subject. Regarding their knowledge about marketing, the majority answered that they had basic knowledge. Still in relation to knowledge about the theme in question, all academics answered that, if they had already graduated, they would use social networks as marketing strategies disseminating videos and posts, as well as online classes. All academics reported that knowledge about marketing is extremely important for the profession. The conclusion is that marketing is being approached and used timidly by the future Physical Education professionals surveyed, and a greater investment in the area by such professionals is necessary.
\end{abstract}

KEYWORDS: physical education. marketing. entrepreneurship. initial education.

\footnotetext{
${ }^{1}$ Acadêmico de bacharelado em Educação Física da Universidade de Cruz Alta.

${ }^{2}$ Professora Mestre do Curso de bacharelado em Educação Física da Universidade de Cruz Alta.

${ }^{3}$ Professora Dra . do curso de bacharelado em Educação Física e do Programa de Pós-Graduação em Atenção Integral á Saúde da Universidade de Cruz Alta.

${ }^{4}$ Professora $\mathrm{Dr}^{\mathrm{a}}$. do curso de bacharelado em Educação Física e do Programa de Pós-Graduação em Atenção Integral á Saúde da Universidade de Cruz Alta.
} 


\section{Introdução}

As plataformas digitais vêm ganhando espaço e notoriedade em tempos recentes, são ferramentas que facilitam o acesso de boa parte da população em diversos meios e serviços. Com avanço da internet e os meios eletrônicos criou-se uma grande alteração no comportamento social dos indivíduos consumidores, desta forma aumenta cada vez mais a busca de meios digitais e mídias sociais (DRUCKER, 2000).

Segundos dados do Instituto Brasileiro de Geografia e Estatística (IBGE, 2018) mais de $69,3 \%$ da população brasileira possuem internet em suas residências, ou seja, mais de 41 milhões de brasileiros tem acesso a internet e utilizam as redes sociais com a finalidade de enviar mensagens, interagir socialmente, trabalhar, estudar, aprender culinária e outras coisas, e saber sobre atividades físicas.

Neste universo das redes sociais houve uma crescente procura por uma vida mais saudável, hábitos alimentares e pratica de exercícios físicos ganhou destaque no mundo das redes sociais. Acompanhando todo esse crescimento o mercado fitness não é diferente, as plataformas digitais vêm ganhando muitos adeptos principalmente por pessoas que não tem muito tempo para treinar, pessoas querem fugir das academias superlotadas, por tanto, as formas de abordagens e de como se trabalhar tiveram suas mudanças para captações de potenciais clientes (STRAUS; FROST, 2012).

O uso de internet se tornou ainda mais importante em época de pandemia da COVID$19^{\mathrm{a}}$ (COELHO et al., 2020). Desde dezembro de 2019 a população mundial, vem sofrendo com o novo coronavirus (SARS- CoV-2) responsável pela pandemia da COVID-19 (ROSER et al., 2020), situação está que limitou muitas atividades da população em geral, tornando as atividades mais restritas, centralizadas em sua própria residência evitando aglomerações.

Com aumento de casos do coronavírus, as academias sofreram um impacto muito grande na sua economia, devido as restrições de funcionamento. Neste sentido, o mercado fitness teve diversos postos de trabalho fechados, demissões em grande escala. Assim, com a dificuldade pela busca do seu primeiro emprego após a sua graduação, os profissionais recémformados ingressaram fortemente na criação do seu próprio negócio como alternativa de trabalho, tais como consultorias online, home fitness, etc. (Conselho Nacional de Educação Física, 2020)

Com a necessidade de manter o distanciamento para frear a diminuição do novo coronavírus, utilização dos meios digitais ganhou força, com isso o mercado digital se tornou uma excelente opção para os futuros profissionais que visam empreender, e mostrar o seu 
profissionalismo criando meios de comunicação onde consegue atender um número maior de potenciais clientes, com um custo de operação muito abaixo de mercado comparado a empresários donos de academias que tem seus custos de ter um empreendimento físico mensalmente (HENRIQUE, 2020).

Nota se que cada vez mais os profissionais de Educação Física precisam estar atentos, preparados e qualificados para o mercado de trabalho, e com isso tais métodos de trabalho como o meio digital vem surgindo como grandes opções, bem como estratégias para atingir o seu público (PAULO, 2015).

Neste sentido, torna-se muito importante saber como o tema marketing está sendo relacionado com as tecnologias digitais e como está sendo abordado na graduação em Educação Física bacharelado (SPACOV, 2016).

Para Kotler e Armstrong (2014) podemos definir marketing como um processo de “atração, conversão e retenção de clientes por meio da geração de um valor sobre produto, serviço ou marca". É o processo pelo qual diversos ramos de negócios criam estratégias para obtenção de renda através de seu trabalho, criando um processo de obtenção de troca de valores.

Sendo assim, este estudo objetivou analisar como a temática marketing digital está sendo estudada, percebida e desenvolvida por acadêmicos de Educação Física Bacharelado.

\section{Métodos}

Esta pesquisa do tipo qualitativa descritiva ocorreu no segundo semestre de 2020 com 10 acadêmicos do curso de Educação Física Bacharelado da Universidade de Cruz Alta (UNICRUZ). Todos estavam matriculados no $8^{\circ}$ semestre do referido curso. Como requisito para participação na pesquisa, só foram convidados a participar da mesma, alunos matriculados no semestre de 2020/2 e que já tivessem cursado a disciplina de Gestão em Marketing Esportivo a grade curricular (2014/1) do curso (PPC, 2015).

A UNICRUZ é uma Instituição de Ensino Superior Comunitária e se localiza no munícipio de Cruz Alta, na região noroeste do Rio Grande do Sul, Brasil.

Como instrumento de pesquisa foi aplicado de maneira digital, via google formulários, enviado por e-mail e por aplicativo WhatsApp, um questionário elaborado pelo próprio pesquisador contendo questões abertas sobre idade; local de residência; bolsa de estudos; realização/tempo de estágio em locais de atuação da Educação Física Bacharelado e o uso do marketing nestes locais; disciplinas da graduação que abordavam o tema de pesquisa; interesse e nível de conhecimento do aluno sobre marketing; e, importância do marketing para 
futura profissão. Para coleta de dados primeiramente contatou-se a coordenadora do curso solicitando autorização para a realização da pesquisa. Posteriormente entrou-se em contato com os alunos matriculados no $8^{\circ}$ semestre do curso para explicação dos objetivos e convite para participar da pesquisa. Os que aceitaram assinaram por meio eletrônico o Termo de Consentimento Livre e Esclarecido (TCLE). Logo após foi aplicado o instrumento de pesquisa.

Os dados da pesquisa foram coletados no período de 15/09/2020 a 15/10/2020. Estes meses foram escolhidos para a condução da pesquisa em função de estar dentro do período letivo onde os acadêmicos desenvolvem suas atividades na graduação.

Os dados foram interpretados por análise de conteúdo e, para manter o anonimato, os acadêmicos foram identificados como $A 1, A 2, A 3$ e assim sucessivamente conforme sugere Minayo (2008).

\section{Resultados e discussão}

Os 10 acadêmicos participantes deste estudo estavam todos no $8^{\circ}$ semestre do curso de Educação Física bacharelado, tinham entre 20 e 33 anos de idade, somente dois não possuíam algum tipo de bolsa de estudos, seis residiam em Cruz Alta, um em Júlio de Castilhos, um em Santa Bárbara do Sul, outro em Ibirubá e um em Panambi.

Todos os participantes relataram que somente a disciplina de Marketing abordou sobre o tema do presente estudo. As outras disciplinas do curso de Educação Física bacharelado não abordaram sobre marketing digital. Neste sentido, a maioria dos alunos (7) disseram que o curso não ofereceu uma boa formação na temática e indicaram ser necessário mais disciplinas que abordem sobre marketing. Eles disseram: "falta mais matérias sobre isso" (A5); "pouco conteúdo" (A6); “...poderia ter ofertado mais conteúdos” (A7); “ofertou somente o básico” (A9).

Flores et al. (2019) pesquisaram acadêmicos em fase de conclusão do curso de Educação Física por meio de grupo focal e evidenciaram que a formação inicial, ou seja, a graduação é muito importante para a futura profissão. Esta formação inicial é influenciada por diversos fatores como a individualidade do sujeito, a participação em ações formativas proporcionadas no decorrer de sua formação inicial e as experiências de vida a partir das relações interpessoais que os motivam a seguir na profissão escolhida.

Neste sentido, o fato de os acadêmicos relatarem que o curso não ofereceu uma boa formação sobre marketing devido a somente uma disciplina abordar a temática é injustificada, 
pois os resultados de Flores et al. (2019) mostram que a formação inicial do acadêmico de Educação Física é muito influenciada pela busca individual pelo conhecimento.

A atualização do profissional de Educação Física precisa ser constante em modernização e inovação nas modalidades ofertadas, com isso pontua-se a importância de utilizar o marketing para a divulgação dos serviços ofertados (SANTOS; NASCIMENTO, 2019).

Os três alunos que relataram que o curso de Educação Física ofereceu uma boa formação sobre marketing, disseram: "ofereceu oportunidades (A3); “mostrou a importância de conhecer mais sobre o assunto (A8); "assim fiquei mais atento no marketing da minha academia" (A10). Estes mesmos três alunos relataram que esta temática despertou o interesse deles como futuros profissionais, sendo que dois realizaram algum curso específico sobre o tema.

Além de vários conhecimentos adquiridos na graduação, o profissional de Educação Física precisa estar em constante aperfeiçoamento de informações de teorias e práticas inovadoras, buscando a realização de cursos, participação em eventos, palestras e workshops, ou seja, manter uma formação continuada, para apropriar-se de conhecimentos, buscando embasamento científico atualizado (BRANCO, 2015).

Todos os acadêmicos percebem que conhecimentos sobre marketing são de extrema importância para a profissão. As falas dos acadêmicos estão no quadro 1 .

Quadro 1: Percepção da Importância do conhecimento sobre marketing na opinião de acadêmicos de Educação Física bacharelado. Cruz Alta, Rio Grande do Sul, Brasil, 2020.

\begin{tabular}{|c|l|}
\hline Acadêmico & \multicolumn{1}{|c|}{ Fala } \\
\hline A1 & após a graduação vai precisar de noção para divulgar seu trabalho \\
\hline A2 & Somos nossa própria empresa, saber gerir, criar e inovar é fundamental \\
\hline A3 & devido ao contexto que estamos \\
\hline A4 & porque faz parte do mundo dos negócios \\
\hline A5 & Questão de visão que os clientes terão sobre nosso trabalho \\
\hline A6 & saber empreender e unir estratégias com o marketing vamos longe \\
\hline A7 & É necessário ter um norte de como gerir um negócio... \\
\hline A8 & é com divulgação da profissão que podemos crescer \\
\hline A9 & $\begin{array}{l}\text { trabalho com lutas, estou a todo momento buscando novos patrocínios e } \\
\text { alunos }\end{array}$ \\
\hline A10 & está colocando em prática todas as técnicas adquiridas na graduação \\
\hline
\end{tabular}


Ao serem abordados sobre o nível de conhecimento que eles (acadêmicos) acreditavam possuir sobre marketing, um relatou não possuir nenhum conhecimento, seis responderam que tiverem conhecimento básico, dois consideraram ter conhecimento intermediário e um avançado.

Ainda em relação ao conhecimento sobre a temática em questão, os acadêmicos foram indagados sobre quais estratégias de marketing eles utilizariam se fossem profissionais de Educação Física formados, levando em consideração este momento difícil que estamos vivendo por causa da pandemia COVID19. Todos (10) responderam que utilizariam marketing digital por meio de redes sociais divulgando vídeos e postagens, além de aulas online.

Em uma sociedade em que o conhecimento tecnológico é relevante no dia a dia, a internet tornou-se muito mais dinâmica no âmbito empresarial, onde as ações criadas online obtêm um feedback dos consumidores em tempo real, podendo ser utilizado como um termômetro para medir a aceitação do público, aperfeiçoando assim os conteúdos disponibilizados (BENETTI, 2020).

Como acentuado por Benetti (2020), as postagens realizadas pelos profissionais de Educação Física em momentos de execução de exercícios e até de momentos de aulas, podem proporcionar um feedback mais rápido de outras pessoas que tenham interesse em conhecer o trabalho desenvolvido. Além disso, o uso cada vez maior de aplicativos de mensagens privadas é uma das tendências do marketing digital em 2020 (VALLE, 2020).

Relacionando a temática com a futura área de atuação destes acadêmicos, indagou-se sobre os estágios voluntários ou obrigatórios, e quais estratégias de marketing estes locais onde eles estagiariam utilizavam. A grande maioria destes locais (8) utilizavam as suas próprias redes sociais como estratégia de marketing, um local fazia divulgações em propagandas em geral e um realizava pesquisa de mercado contratando empresas de consultoria. Evidenciando assim, mais uma vez, a importância do marketing digital na área de Educação Física, como já discutido e embasado anteriormente.

Este período de pandemia da COVID-19 fez com todas as profissões mudassem suas estratégias de marketing, utilizando o meio digital de diferentes maneiras (COSTA; FIGUEIREDO, 2020; PINTO et al., 2020). Na Educação Física isso também ocorreu (VALLE, 2020). 


\section{Conclusão}

Evidenciou-se no presente estudo que a temática marketing digital está sendo estudada, percebida e desenvolvida por acadêmicos de Educação Física Bacharelado. Os acadêmicos relataram que possuíam conhecimento básico sobre o tema e que saber/estudar/conhecer sobre marketing é de extrema importância para a profissão. Eles explicaram que se já fossem formados, utilizariam como estratégias de marketing as redes sociais divulgando vídeos e postagens, além de aulas online. Como ponto negativo dos achados do estudo, os participantes relataram que o curso não ofereceu uma boa formação na temática e indicaram ser necessário mais disciplinas que abordassem sobre marketing tendo em vista que todos os participantes relataram que somente a disciplina de Marketing abordou sobre o tema.

Sendo assim, o marketing é muito importante no processo de divulgação e promoção do trabalho desenvolvido pelos profissionais de Educação Física, onde a sociedade acompanha diariamente as postagens, interessando-se e buscando praticar determinadas atividades físicas em virtude do "marketing influencer" provocado de modo online.

O conhecimento de diferentes ferramentas para publicação das modalidades desenvolvidas é importante para dar mais visibilidade ao profissional no mercado, no qual estão disponíveis inúmeras ferramentas que podem ser utilizadas de modo gratuito e também aquelas que podem se impulsionar a publicação atingindo mais pessoas e conseguindo assim visualizar o retorno sobre investimento, tendo uma visão mais ampla de suas ações no meio de marketing digital.

Acredita-se que este estudo pode auxiliar em futuras pesquisas sobre marketing e influenciar alunos de graduação em Educação Física a pensar/estudar/pesquisar mais sobre o tema.

\section{REFERÊNCIAS BIBLIOGRÁFICAS}

AMA - American Marketing Association. Disponível em:

https://www.ama.org/search/pages/results.aspx?k=marketing. Acesso em: 2 jul. 2020.

BENETTI, R. Marketing Digital em 2020: o que é e como funciona? Disponível em: $<$ https://www.organicadigital.com/blog/afinal-como-funciona-o-marketing-digital/> . Acesso em: 3 dez. 2020.

BRANCO, A. E. Ginástica Laboral: Prerrogativa do Profissional de Educação Física. Rio de Janeiro: CONFEF, 2015.

CILETTI, D. Marketing Pessoal. São Paulo: Cengage Learning, 2011. 
COELHO, A. L. et al. A utilização de tecnologias da informação em saúde para o enfrentamento da pandemia do Covid-19 no Brasil. Cadernos Ibero-Americanos de Direito Sanitário, v. 9, n. 3, p. 183-199, 2020.

CONSELHO FEDERAL DE EDUCAÇÃO FÍSICA (CONFEF). ofício nº 370/2020. Rio de janeiro: 2020.

CORTEZ, E.V. A Magia do Marketing Pessoal: O segredo das pessoas bem sucedidas. São Paulo: Alaúde, 2004.

COSTA, M. M. C.; FIGUEIREDO, G. L. A. F. ESTRATÉGIAS DE MARKETING EM MEIO À PANDEMIA. Facit Business and Technology Journal, v. 1, n. 21, 2020.

COSTA, N.E. Marketing Pessoal: uma abordagem para agentes do mercado imobiliário. Goiânia: AB, 2002.

DRUCKER, P. Além da Revolução da Informação. In: HSM Management 18, p. 4855, jan./fev. 2000 RAMOS, J. J. Os Exercícios Físicos na História e na Arte do Homem Primitivo aos nossos dias. São Paulo. Ibrasa, 1982.

FERREIRA JUNIOR, Achiles Batista; AZEVEDO, Ney Queiroz. Marketing digital: uma análise do mercado 3.0. Curitiba: Intersaberes, 2015.

FLORES, P. P. et al. O processo de identização docente em um curso de Educação Física na perspectiva de seus discentes. Journal of Physical Education, v. 30, e3075 2019. Acesso em: 12 dez. 2020.

GABRIEL, Martha. Marketing na era digital, conceitos, plataformas e estratégias. São Paulo: Novatec, 2010.

HENRIQUE, D. C.; CUNHA, S. K. Metodologias, Recursos e Práticas Didáticopedagógicas no Ensino de Empreendedorismo em Cursos de Graduação e Pós graduação Nacionais e Internacionais. In: Encontro Nacional de Programas de Pósgraduação em Administração, p.1-16. 2006. Acesso em: 12 dez. 2020.

HENRIQUE, Trazíbulo. COVID-19 e a internet (ou estou em isolamento social físico). Interfaces Científicas - Humanas e Sociais, Aracaju, v. 8, n. 3, p. 173-176, 2020.

IBGE - INSTITUTO BRASILEIRO DE GEOGRAFIA E ESTATISTICAS. Brasília, 2018, Disponível em: $<$ https://agenciadenoticias.ibge.gov.br/agencia-sala-de-imprensa/2013agencia-de-noticias/realese/20073-pnad-continua-tic-2016-94-2-das-pessoas-que-utilizam-ainternet-o-fizeram-para-trocar-menssagem>. Acesso em: 14 dezembro de2020.

KOTLER, P. ARMSTRONG, G. Marketing Esportivo: Princípios de Marketing. 15 Ed. São Paulo: Pearson Prentice Hall, 2014.

MINAYO, M. C. S. O Desafio do conhecimento: pesquisa qualitativa em saúde. 11 ed. São Paulo: Hucitec, 2008. 
MINISTÉRIO DA SAÚDE. Gabinete do Ministro. Portaria ${ }^{\circ} 188$, de 3 de fevereiro de 2020. Diário Oficial da União 2020; Acesso em: 12 dez. 2020. Disponível em: Portaria-188-20-ms (planalto.gov.br).

OLIVEIRA, Djalma de Pinho Rebouças de. Empreendedorismo: Vocação, Capacitação e Atuação direcionadas para o plano de negócios. São Paulo: Atlas, 2014.

ORGANIZAÇÃO PAN-AMERICANA DA SAÚDE. OMS declara emergência de saúde pública de importância internacional por surto de novo coronavírus. Disponível em: OPAS/OMS Brasil - OMS declara emergência de saúde pública de importância internacional por surto de novo coronavírus (paho.org). Acesso em: 12 dez. 2020.

PAULO, L. F. DE L. Treinamento desportivo - da saúde e emagrecimento ao alto rendimento. Timburi/SP: Cia do ebook, 2015.

PARADINI. Estudo do potencial empreendedor dos acadêmicos no primeiro período do curso de educação física, 2009.

PINTO, Pâmela Araujo et al. COVID-19 no Instagram: práticas de comunicação estratégica das autoridades de saúde durante a pandemia. Comunicação Pública, v. 15, n. 29, 2020.

PROJETO PEDAGÓGICO DO CURSO DE EDUCAÇÃO FÍSICA (PPC). Renovação: Portaria $n^{\circ}$ 1096/15 - 24/12/2015. DOU: 30/12/2015. Disponível em:

$<$ https://home.unicruz.edu.br/wp-content/uploads/2019/10/PPC-Educação-Física-

Bacharelado-2019.pdf>. Acesso em: 16 dez. 2020.

ROSER, M. et al. Coronavirus Pandemic (COVID-19). Publicado online em OurWorldInDate.org. Disponível em: Coronavirus Pandemic (COVID-19) - Statistics and Research - Our World in Data. Acesso em: 14 dez. 2020.

SANTOS, P.V.R; NASCIMENTO, O.V. O Personal Trainer: Estratégias de Marketing para Retenção de Clientes. Revista Campo do Saber, v. 4, n. 22019.

STRAUSS, J.; FROST, R. E-Marketing. São Paulo: Pearson Prentice Hall, 2012.

VAZ, Gil Nuno. Marketing Institucional: O mercado de Ideias e Imagens. São Paulo: Pioneira, 1995.

SILVA, Francisco Martins da. Recomendações as condutas e procedimentos do profissional de educação física na atenção básica de saúde. Rio de Janeiro: CONFEF, 2010.

SPACOV, Y.F. O personal trainer: uso de estratégias de marketing na atividade profissional. Dissertação (Mestrado), UNINOVE, 2016.

VALLE, A. Tendências do Marketing Digital em 2020. Disponível em: $<$ https://www.academiadomarketing.com.br/tendencias-do-marketing-digital-em-2020/>. Acesso em: 3 dez. 2020. 